\title{
Performance Analysis of Multihop CC-HARQ over Lognormal Shadowed Nakagami-m Fading Channels
}

\author{
Jun-Mei HAN ${ }^{\mathrm{a}}$, Yong XI and Ji-Bo WEI \\ School of Electronic Science and Computer Engineering, National University of Defence Technology, Changsha, \\ 410073 China
}

\begin{abstract}
The performance of truncated Chase Combining based Hybrid Automatic Repeat reQuest (CC-HARQ) in multihop relay network is analysed. Different from prior works, composite lognormal shadowed Nakagami fading channels are considered. By extending the Moment Generation Function (MGF) method to approximate the output signal-to-noise ratio to a lognormal random variable, the end-to-end outage probability is derived. The accuracy of the proposed approximating method is validated by simulations and the factors to affect such accuracy are thoroughly studied. The results have lots of potential usage in the analysis and design of practical networks such as power allocation, relay placement and optimization for energy efficiency etc.
\end{abstract}

\section{Introduction}

Hybrid Automatic Repeat reQuest (HARQ) protocols are widely employed to increase the reliability and efficiency of data transmission over wireless communication by combining forward errorcorrecting coding (FEC) with ARQ technique. Particularly, the Chase Combining based HARQ (CCHARQ) scheme, in which the same packet is retransmitted in case of errors and combined with maximal-ratio combining (MRC), is widely used for the benefit of its simplicity and efficiency. HARQ schemes have been adopted in many wireless standards, such as High Speed Packet Access (HSPA), IEEE 802.15.4, IEEE 802.11 and Long Term Evolution (LTE). In addition, with the advent of its coverage extension and spatial diversity gain, multihop relay technique has gained considerable interest in the research community. Concepts such as smart home, smart grid and system control and automation have drawn considerable attention from researchers across the globle and it has been shown that multihop relay networks is one of the most promisising solutions for implementing such concepts [1].

Although various schemes have been proposed to analysis the performance of multihop networks or HARQ schemes, most of them considered only pure large-scale fading [1], [2] or small-scale fading [3]. No complete analysis of the performance for multihop networks adopting HARQ retransmission technique under composite shadowing-fading channels has been reported so far.

In this paper, a new approach of outage probability approximation is proposed for performance analysis of multihop CC-HARQ system under general channel conditions. We consider channel randomness from two channel fading components and the channel coefficient follows a composite

\footnotetext{
${ }^{\text {a }}$ Corresponding author: hjm.han@163.com
} 
Nakagami- $m$ /Lognormal distribution, where the small-scale fading from multipath propagation is modelled by a Nakagami- $m$ Random Variable and large-scale fading from shadowing is approximated by a Lognormal random variable. Due to the complexity of Nakagami- $m$ /Lognormal distribution and multihop CC-HARQ as well as the limit of maximum retransmission number, the analysis becomes very challenging.

In the following, first, the outage probability for single-hop CC-HARQ protocols is obtained by extending the Moment Generation Function (MGF) method to approximate the output signal-to-noise ratio (SNR) to a Lognormal random variable, whose cumulative distribution function $(c d f)$ is obtained by numerically solving for its mean and standard deviation. Latter, the results are particularized for the multihop relay networks. The derived expressions are validated by Monte Carlo simulations. Due to the versatile and flexible features of Nakagami- $m$ fading, it is worthy to note that the Nakagami$m$ /Lognormal channel has the advantage of including the Rayleigh-Lognormal and RicianLognormal channels as special cases.

\section{CC-HARQ over Nakagami-m/Lognormal channels}

We begin by extending the MGF-based approximating method to approximate the output SNR of Nakagmai- $m$ /Lognormal fading with CC-HARQ, and the Gaussian-Hermite integral quadrature rule $[4$, formula (25.4.46)] is applied to approximately reduce the infinite nested integral to a finite number of weighted summations of Hermite polynomials. Then the tightness of the adopted approximation are studied and we examine performance scaling with respect to the SNR thresholds $\gamma_{\text {th }}$ the Hermite polynomial order $N_{P}$ and the maximum retransmission $L$.

We adopt truncated CC-type HARQ in the linear $K$-hop relay network with maximum retransmission number $L$ over Nakagmai- $m$ /Lognormal channels. The relay nodes purely help to forward the information bits from the transmitter to receiver, with half-duplex mode. We further assume that the $L$ packets for the same hop suffer identically distributed and exponentially correlated Nakagami- $m$ /Lognormal channels. The corresponding channel correlation matrix $\mathbf{R}$ is in a Hermitian Toeplitz structure, with the $x$-th column and $y$-th row element $\mathbf{R}_{l}(x, y)=\eta_{l}^{|x-y|}, \eta_{l} \in[0,1]$. For different hops $R_{i} \neq R_{j}$, the channel gains $h_{i}$ and $h_{j}$ are mutually independent and may assume different fading models. The instantaneous SNR of the $k$-th hop of $l$-th transmission is $\gamma_{k_{l}}=\left(P_{k_{l}} / N_{0}\right)\left|h_{k_{l}}\right|^{2}$, where $N_{0}$ is identical power of the additive Gassian white noises.

\subsection{The Single hop outage probability}

For the k-th hop, the output SNR in point-to-point transmission is the sum of L individual retransmission $\left(\gamma_{k}=\sum_{l=1}^{L} \gamma_{k_{l}}\right)$. The desired outage probability, which is basically equal to the $c d f$ of output SNR at a specific threshold $\gamma_{t h}$, can be expressed as:

$$
\begin{aligned}
& P_{\text {out }}\left(\gamma_{\text {th }}\right)=\operatorname{Pr}\left\{\gamma_{\text {MRC }} \leq \gamma_{\text {th }}\right\} \\
& =\operatorname{Pr}\left\{\sum_{l=1}^{L} \gamma_{k_{l}} \leq \gamma_{t h}\right\}=\operatorname{Pr}\left\{\gamma_{k} \leq \gamma_{t h}\right\} .
\end{aligned}
$$


The joint composite distribution of received SNR $\left(\gamma_{k}\right)$ for all the $L$ retransmission can be obtained by averaging the joint Nakagami-m distributed instantaneous SNR over the conditional density of the joint Lognormal distributed average SNR, whose $p d f$ is given by :

$$
p_{\gamma_{k}}\left(\gamma_{k}\right)=\int_{0}^{\infty} p_{\gamma_{k} \mid \bar{\gamma}_{k}}\left(\gamma_{k} \mid \bar{\gamma}_{k}\right) p_{\bar{\gamma}_{k}}\left(\bar{\gamma}_{k}\right) d \bar{\gamma}_{k}
$$

Note that (2) is composed of two components. The first component, i.e., the $p d f$ of $\gamma_{k}$ conditioned on the average SNR $\bar{\gamma}_{k}$, arises from multipath fading and is given by a Gamma distribution $f(\cdot)$ (Generally, $f(x ; k, \phi)$ denotes the $p d f$ of Gamma distribution with the shape parameter $k$ and the scale parameter $\phi$, given by: $f(x ; k, \phi)=\frac{1}{\phi^{k}} \cdot \frac{x^{k-1}}{\Gamma(k)} \cdot \exp \left(-\frac{x}{\phi}\right)$, where $\Gamma(\cdot)$ is the Gamma function). Let $m_{k}$ denote the fading severity measure. The first component can be written as:

$$
p_{\gamma_{k} \mid \bar{\gamma}_{k}}\left(\gamma_{k} \mid \bar{\gamma}_{k}\right)=f\left(\gamma_{k} ; \frac{m_{k} L^{2}}{u_{k}}, \frac{u_{k} \bar{\gamma}_{k}}{m_{k} L}\right)
$$

with $u_{k}=L+\frac{2 \eta_{k}}{1-\eta_{k}}\left(L \frac{1-\eta_{k}^{L}}{1-\eta_{k}}\right) \cdot \mu_{k}(\mathrm{~dB})$ and $\sigma_{k}(\mathrm{~dB})$ are the mean and standard deviation of $10 \log _{10} \bar{\gamma}_{k}$, respectively.

The second component of (3), $p_{\bar{\gamma}}(\bar{\gamma})$, is the joint $p d f$ of average SNRs over all retransmission packets. The randomness of each average SNR is affected by the shadowing and is approximated by a Lognormal random variable:

$$
p_{\bar{\gamma}_{k}}\left(\bar{\gamma}_{k}\right)=\frac{\lambda}{\sqrt{2 \pi} \bar{\gamma}_{k}} \cdot \exp \left[-\frac{1}{2}\left(10 \log _{10} \bar{\gamma}_{k}-\mu_{k}\right)^{T}\left(10 \log _{10} \bar{\gamma}_{k}-\mu_{k}\right)\right]
$$

where $\lambda$ is the scaling constant $(\lambda=10 / \ln 10=4.3429)$. By substituting $\omega_{k}$ for $\left(10 \log _{10} \bar{\gamma}_{k}-\mu_{k}\right) / \sqrt{2} \sigma_{k}$, we obtain a simplified expression for the joint composite $p d f$,

$$
p_{\gamma_{k}}\left(\gamma_{k}\right)=\frac{1}{(\sqrt{\pi})} \int_{-\infty}^{\infty} f\left(\gamma_{k} ; \frac{m_{k} L^{2}}{u_{k}}, \frac{u_{k} \cdot g\left(\omega_{k}\right)}{m_{k} L}\right) e^{\left(-\omega^{T} \omega\right)} d \omega
$$

Where $g\left(\omega_{k}\right)=10^{0.1\left(\sqrt{2} \sigma_{k} \omega_{k}{ }_{k}\right)}$.

Due to the nested integral form, the closed-form $m p d f$ expression for the composite Nakagami$m$ /Lognormal fading distributions is hard to get. However, it has been illustrated that the sum of composite Rayleigh-Lognormal fading random variables can be approximated by a Lognormal random variable by [5] and [6]. We extend those work to approximate the output SNR of Nakagami- 
$m /$ Lognormal fading with CC-HARQ to a new Lognormal random variable. Therefore, we can calculated the outage probability with only the means $\left(\mu_{\Gamma}\right)$ and standard derivation $\left(\sigma_{\Gamma}\right)$ of the new Lognormal random variable, which reduced the computation complexity significantly. The $p d f$ of the new random variable takes the form:

$$
p_{\Gamma}\left(\gamma^{\prime}\right)=\frac{\lambda}{\sqrt{2 \pi} \sigma_{\Gamma} \gamma^{\prime}} \exp \left[-\frac{\left(10 \log _{10} \gamma^{\prime}-\mu_{\Gamma}\right)^{2}}{2 \sigma_{\Gamma}^{2}}\right]
$$

We resort to an MGF-based approximating method to estimate the Lognormal characterized parameters $\mu_{\Gamma}$ and $\sigma_{\Gamma}$. Specifically, the corresponding parameters are estimated by matching respective MGFs at two different, real and positive value of $S$, namely, $s_{1}$ and $s_{2}$. This method consists of two independent equations as follows:

$$
\Psi_{\Gamma}\left(s_{k}\right)=\Psi_{\gamma_{C C}}\left(s_{k}\right), k=1,2 .
$$

The Gaussian-Hermite integral quadrature rule is applied to approximately reduce the infinite integral to a finite number of weighted summations of Hermite polynomials. Accordingly, $\mu_{\Gamma}$ and $\sigma_{\Gamma}$ of the new Lognormal random variable $\Gamma$ can be readily calculated. Through extensive derivations, the exact relationship between the original parameters and approximated one can be obtained as:

$$
\left\{\begin{array}{l}
\mu_{k^{\prime}}=\mu_{k}+\lambda\left[\psi\left(m_{k}\right)-m_{k}\right] \\
\sigma_{k^{\prime}}=\sqrt{\lambda^{2} \varsigma\left(2, m_{k}\right)+\sigma_{k}^{2}}
\end{array}\right.
$$

Where $\varsigma\left(2, m_{k}\right)=\sum_{i=0}^{\infty} \frac{1}{\left(m_{k}+i\right)^{2}}$ is the Reimann's Zeta function. $\psi\left(m_{k}\right)=-C+\sum_{i=1}^{m_{k}-1} \frac{1}{i}$ is the Euler Psi function, $C$ is the Euler's constant ( $C \approx 0.5772$ ). The outage probability in (1) can be calculated by: $P_{\text {out }}\left(\gamma_{\text {th }}\right) \cong \int_{0}^{\gamma_{t h}} p_{\Gamma}\left(\gamma^{\prime}\right) d \gamma^{\prime}$.

\subsection{The end-to-end outage probability}

We now investigate the end-to-end outage probability of multihop relay networks which is given by:

$$
\begin{aligned}
& P_{e 2 e}=\operatorname{Pr}\left\{\min \left(\gamma_{1}, \gamma_{2}, \ldots, \gamma_{K}\right) \leq \gamma_{t h}\right\} \\
& =1-\prod_{k=1}^{K} \operatorname{Pr}\left\{\gamma_{k}>\gamma_{t h}\right\}=1-\prod_{k=1}^{K} Q\left(\frac{\left(\log \gamma_{k}-\mu_{\Gamma}\right)}{\sigma_{\gamma_{k}}}\right)
\end{aligned}
$$


Where $Q(\cdot)$ is the Gassian Q-function (i.e., $Q(x)=\int_{x}^{\infty} \frac{1}{\sqrt{2 \pi}} e^{-y^{2} / 2} d y$ ). Roughly speaking, the desired outage probability at $\gamma_{t h}$ expressed in (9) is dependent on the retransmission number $L$, the number of hops $M$, the received SNR, and other location specific propagation characteristics of individual branches, e.g., severity measure of multipath fading $m_{l}$, local mean power $\mu_{l}^{\prime}$ and standard deviation of shadowing $\sigma_{l}^{\prime}$.

\section{Analytical and simulation results}

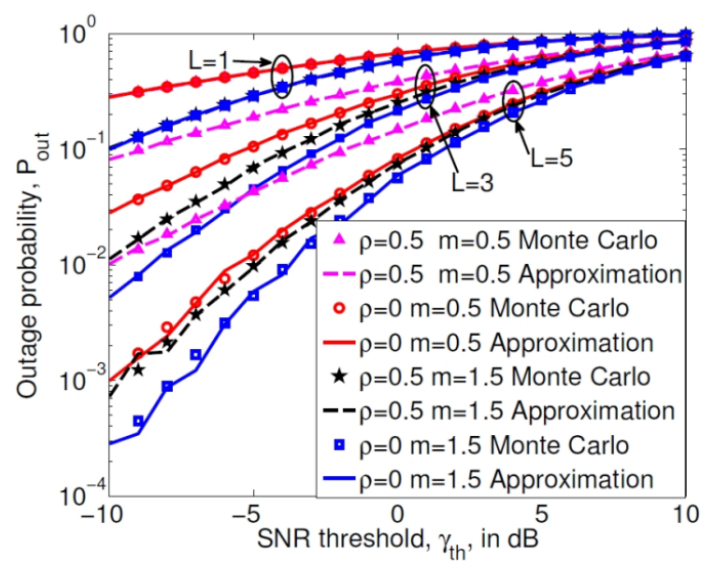

Figure 1. The Outage probability according to the SNR threshold.

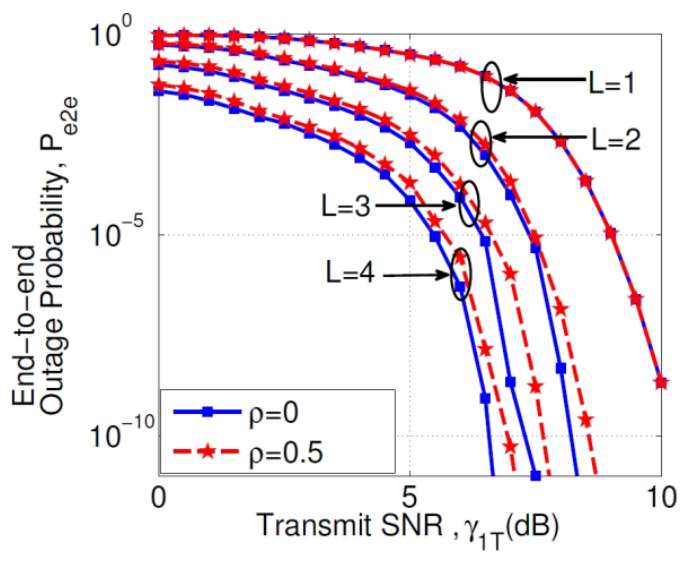

Figure 2. The end-to-end outage probability according to the transmit SNR for $M=3$.

In this section, the simulation and analytical results are presented. Fig. 1 and Fig. 2 show the outage probability performance. Fig. 1 is the outage probability performance of exponential correlation model for single hop CC-HARQ at different SNR thresholds. The same mean path loss and standard deviation of shadowing are assumed to be $\mu=0 \mathrm{~dB}$ and $\sigma_{i}=6 \mathrm{~dB}$. Fig.2 is the end-to-end outage 
probabilities performance, and the results are generated by varying the transmit SNR while maintaining the ratio among individual hop noise power ration $P_{T} / N_{0}$ as $\gamma_{1}: \gamma_{2}: \gamma_{3}=1: 2: 3$. Three hop systems are considered with $\gamma_{t h}=5 \mathrm{~dB}$ and severity measure of multipath fading factors for three hop are $m_{1}=0.5, m_{2}=2, m_{3}=1$. The Monte Carlo simulations with one million samples match with approximation method nicely, thereby validating the accuracy of the algorithm. The figures also display comparisons between two different levels of correlation ( $\rho=0$ or 0.5 ), as well as among different numbers of retransmission ( $L=1,3,7$ ).

As expected, increasing the correlation level $\rho$ degrades the outage performance, while increasing the retransmission number $L$ improves it. The efficient of correlation level $\rho$ is more remarkable in the low $\gamma_{t h}$. This is a consequence of the fact that the approximation underestimation is mainly affected by the fading effect on the composite showing-fading distribution and the showing effect is slighter. In particular, at the higher value of $\gamma_{t h}$, the overall variability of composite showing-fading distribution is mainly cause by the Lognormal showing component, which is relate to transmit distance and is independent to other rounds. In addition, due to the multiplication between fading component and showing component, the disturbance effect of fading is much slighter at the lower value of transmit SNRs.

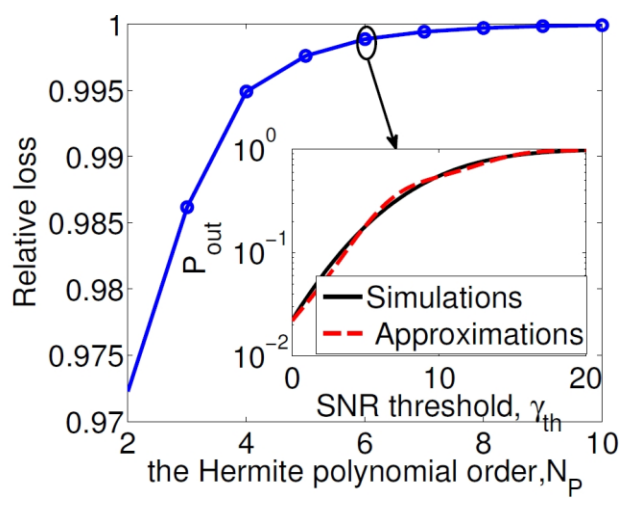

Figure 3. The relative loss of the proposed evaluation approaches under different $N_{P}$ with $L=4$.

The accuracy and efficiency of these approaches are dependent on the maximum retransmission number $L$ and the Hermite polynomial order $N_{p}$. The corresponding parameters for Figure 3 and Figure 4 are as follows unless otherwise stated: $\mu=0 \mathrm{~dB}, \sigma=6 \mathrm{~dB}, \rho=0.5$ and $m=1.5$.

Figure. 3 shows the relative loss for the proposed evaluation approaches $N_{p}$. Note that although the mismatch from the standard value will decrease when $N_{p}$ increases, accurate results can be obtained even for small order of $N_{p}$. Additionally, increasing $N_{P}$ will lengthen the runtime. As shown in the figures, $N_{P}=6$ is the compromising values which guarantee both the accuracy and efficiency. It should be noted that $\mathbf{S}$ should be chosen appropriately to reduce the results mismatch. [5] provides guideline for $\mathbf{S}$ choosing according to the desired outage probability. In our scenarios, we find $\left(s_{1}, s_{2}\right)=(1,0.1)$ provides a good fit as shown in the figures. 


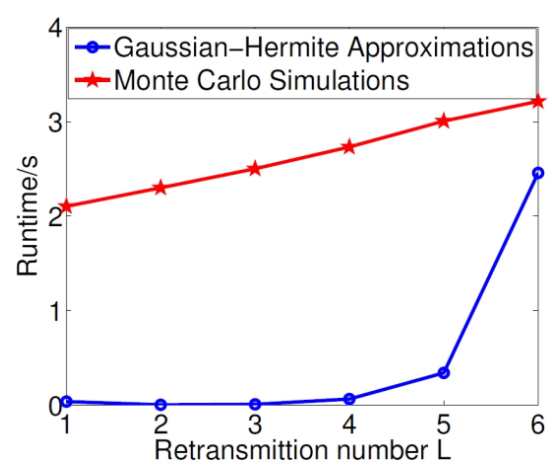

Figure 4. The runtime of the proposed evaluation approaches under different $L$ levels with $N_{P}=6$.

Figure. 4 compares the runtime of the proposed evaluation approach with extensive Monte Carlo simulations for different $L$. The proposed approach is more efficient than the Monte Carlo simulations with one million samples. Note that the runtime is mainly affected by the complexity of the approach, the runtime of Gaussian-Hermite Quadrature is exponentially increases with the $L$. In fact, when $L$ become larger $(L>8$ ), the runtime becomes intolerable (more than 30 seconds). Fortunately, in most practical systems $L$ is usually less than 8 due to the requirement of delay and efficiency.

\section{Conclusion}

This paper has presented a general numerical approach to evaluate the outage probability of multihop relay networks adopting CC-HARQ with $L$ retransmissions under Lognormal shadowed Nakagami$\mathrm{m}$ fading channels. An MGF-based method is proposed to approximate the output SNR by a new Lognormal random variable, whose characterized parameters are numerically solved. Based on the approximation, the outage probability can be calculated by the $c d f$ of this approximating random variable at a specific threshold. This approximation is simple and is proved to be tight enough to address the outage probability. In addition, this evaluation approach is feasible to be used for the optimal design of system parameters, such as the optimal power and rate designs to achieve the maximum delay limited throughput.

\section{References}

1. Ankit.Dubey, Ranjan K.Mallik, Robert.Schober. :'Performance analysis of a multi-hop power line communication system over log-normal fading in presence of impulsive noise', IET Commun., pp.1-9, 9, 1 (2015).

2. Chinmoy. Kundu and Ranjan. Bose,: 'Joint optimal power allocation and relay location for decode-and-forward multi-hop relaying over log-normal channel', IET Commun., pp.2197-2207, 9, 18 (2015).

3. Peng.Wu and Nihar.Jindal.: 'Performance of Hybrid-ARQ in Block-fading Channels: A Fixed Outage Probability Analysis' Wireless Commun. IEEE Trans on, pp. 1129-1141, 58, 4 (2010).

4. Milton Abramowitz and Irene A. Stegun: 'Handbook of mathematical functions with formulas, graphs, and mathematical tables', (1964).

5. Mehta N B, Wu J, Molisch A F, et al.: ' Approximating a Sum of Random Variables with a Lognormal Anderson', Wireless Commun. IEEE Trans on., pp. 2690-2699, 6 (2007). 
6. Rui. Zhang and Jibo.Wei, etc: 'Outage Probability of MRC Diversity over Correlated Shadowed Fading Channels', Lett. IEEE Wireless Commun., pp.516-519, 1, 5 (2012). 